\title{
Should Dental Amalgam Fillings Fill Us with Fear?
}

\author{
Andrea 't Mannetje \\ Centre for Public Health Research, Massey University, Wellington, New Zealand
}

Dental amalgam fillings have been used to reconstruct decayed teeth for nearly two centuries [1]. Amalgam fillings contain mercury which has long been known to be toxic. The phrase 'mad as a hatter' refers to the 19th-century occupational disease that resulted from prolonged contact with mercury used in the manufacture of felt hats. It is therefore not surprising that there has long been a controversy regarding the possible health effects related to amalgam fillings. These health concerns range from subjective health complaints, to neurologic diseases and cancer [2]. The debate on the safety of amalgam fillings is, however, marked by a profusion of badly designed studies, with only a few adequate epidemiological studies that have used objective measures of exposure and health and could exclude major bias. An example of a well designed study is a retrospective cohort [3] including 20,000 people in the New Zealand Defence Force who all received regular dental treatment from the army dental service. Individual records on amalgam filling placements were available. The cohort was followed up from 1977 to 1997 and linked with cancer registrations and hospital admissions. Hazard ratios (risk associated with 100 amalgam-filled-surface-years, relative to the risk associated with having no amalgam exposure) for all major disease categories were presented. This study showed a hazard ratio of 1.00 (95\% Confidence Interval: 0.97-1.03) for cancer, based on 264 cancer cases, indicating no association between amalgam fillings and cancer.

In this issue of ONKOLOGIE, the author of a study of 114 cancer cases with dental amalgam fillings [4], concludes that exposure to amalgam could play an important role in the development of cancer. This was based on his observation that younger cancer cases had their first amalgam filling at an earlier age than cases diagnosed at an older age, which led him to conclude that early exposure to amalgam fillings could cause the development of cancer at an earlier age. He shows that the association is statistically significant, and that it is present for all cancers combined, as well as for all specific cancer sites that were studied.

How should we interpret this? Why did this study observe a strong association while another larger and well designed study did not? The answer can be found in an epidemiological bias known as the 'cohort effect'. The observed association is easily explained by this phenomenon, and is most likely unrelated to cancer.

A 'cohort effect' is a phenomenon where the birth cohorts (generations) within a study population, differ from each other for some factor (e.g. dental health). Cohort effects can severely confuse the interpretation of cross-sectional data. An illustrative example of this bias is the finding that left-handed individuals die younger than right-handers. Because left-handedness was strongly discouraged in older birth cohorts, lefthandedness is more common in younger birth cohorts. When using cross-sectional data, the proportion of left-handers will therefore be larger among those who die at 40 than among those who die at 80 , resulting in a younger average age of death for the left-handers. This illustrates that causal inferences can not be trusted if they depend on comparisons of average age between people with different characteristics [5]. The study by Müller [4] included patients diagnosed with cancer at various ages during the study period 1990-1996. The study subjects of different ages therefore come from different birth cohorts, with the oldest born in 1928 and the youngest in 1969. Over this time period, marked changes in oral health have occurred, resulting from changes in dental care, access to dental care, sugar consumption, fluoride availability and other preventive programmes. All these factors have influenced the prevalence of dental amalgam fillings in the population, and the average age of receiving the first amalgam filling. Comparing different birth cohorts will therefore inevitably show differences in markers of dental health. It is plausible that younger generations, although having a better oral health

\begin{tabular}{ll}
\hline KARGER & ( 2006 S. Karger GmbH, Freiburg \\
Fax +497614520714 & Accessible online at: \\
$\begin{array}{l}\text { E-mail Information@Karger.de } \\
\text { www.karger.com }\end{array}$ & www.karger.com/onk \\
&
\end{tabular}

Andrea 't Mannetje, $\mathrm{PhD}$

Centre for Public Health Research

Massey University

PO Box 756

Wellington, New Zealand

E-mail a.mannetje@massey.ac.nz 
overall, had their first amalgam filling at a younger age than older generations because of increased sugar consumption and earlier detection of caries because of improved dental care. This may well explain why the younger study subjects had their first filling at an earlier age on average than the older study subjects. The author interpreted this as a temporal association between the age at receiving the first amalgam filling and the age of developing cancer. In fact, this association is likely to be present in any study population consisting of the same birth cohorts, i.e. we would see the same pattern in a random sample of people without cancer. This hypothesis can not be confirmed without additional data, but is supported by the lack of organ specificity of the findings. In occupational studies with exposure levels to mercury far higher than that could result from dental amalgam, the main target organs of mercury are the brain and kidney [6]. In the study of Müller the association was observed for all studied cancer sites, including cancer of the colon, breast, reproductive organs, and a group of other cancers. The lack of organ specificity of the association therefore argues against a causal association between age of first dental amalgam and the development of cancer, and is consistent with the above described 'cohort effect' as a plausible explanation for the observed association.

The above example illustrates that poorly designed studies and uncritical evaluation of their results can lead to a faulty interpretation of the association between dental amalgam and health effects. In face of the current evidence, how then should we answer the question posed in the title? A recent review of the few relevant studies [2] does not support an association between dental amalgams and cancer. Neither are renal effects or ill-defined symptom complexes such as chronic fatigue symptoms likely caused by amalgams [2]. Some studies have however suggested a small increased risk for multiple sclerosis [3], and further studies are needed into neurologic diseases, reproductive hazards and effects in children [2]. Any new research should, however, involve well-designed cohort or case-control studies that avoid the bias inherent in the study of Müller.

\section{References}

1 Clarkson TW: The three modern faces of mercury. Environ Health Perspect 2002;110 (suppl 1):11-23.

2 Bates MN: Mercury amalgam dental fillings: An epidemiologic assessment. Int J Hyg Environ Health 2006. [epub ahead of publication]

3 Bates MN, Fawcett J, Garrett N, Cutress T, Kjellstrom T: Health effects of dental amalgam exposure: a retrospective cohort study. Int J Epidemiol 2004;33: 894-902.

4 Müller WHO: Dental Amalgam Fillings and Cancer - Consideration of LongTerm Processes. Onkologie 2006;29:227-230.

5 Peto R: Left handedness and life expectancy. Causal inferences cannot be trusted. BMJ 1994;308:408.

6 IARC Monographs on the evaluation of carcinogenic risks to humans: IARC Monographs Vol. 58: Mercury and mercury compounds, 1993. 